\title{
Biological agents in asthma therapy: An overview
}

\author{
Yalcin AD * \\ Department of Internal Medicine, Allergy and Clinical Immunology, Antalya Education and Research Hospital, \\ Antalya, Turkey
}

\section{Editorial}

Biotechnological ideas are getting bigger and bigger nowadays. As more and more innovator/inventor choose treatment as convenient way of therapy, biologic agents and other facilities valuable methods must be discovered in order to cope with a growing number of biological agents. This special issue emphasize on new generation monoclonal humanized antibodies in asthmatics [1-3].

The pathophysiological mechanisms underlying asthma which is a heterogeneous disease are characterized by interactive responses among various cell types and the hematopoietic cells of the adaptive and innate immune systems. In addition, humanized monoclonal antibodies (HMA) evaluated for the treatment of severe persistent asthma (SPA) but not approved after Phase II trial are as follows; Infliximab (Recombinant human-murine chimeric anti-TNFa monoclonal antibody Infliximab), Etanercept (Soluble TNFa receptor fusion protein), Golimumab (Fully human TNFa-blocking antibody), MEDI-528 (Humanized IgG1 monoclonal antibody that binds to IL-9) [4].

Approved HMA for the treatment of SPA in Phase II/III studies are as follows; Quilizumab (directed against an extracellular 52-aminoacid segment termed M1 prime of human membrane IgE through reduction of new IgE-producing plasma cells), Ligelizumab (Binds C3 domain of IgE), Dupilumab (Binds IL-4Ra inhibiting both IL-4 and IL-13 signaling), AMG 317 (AMG 317 is a fully human monoclonal IgG2 antibody to IL-4R), AMG 157(Human anti-TSLP monoclonal immunoglobulin G2k that specifically binds human TSLP), Pitrakinra (Recombinant human IL-4 variant that is a potent inhibitor of both the IL-4 and IL-13 receptors), Altrakincept (Soluble recombinant human IL-4 receptor: Phase III study completed), Pascolizumab (Humanized mAb blocking IL-4), Tralokinumab (IL-13-specific human monoclonal antibody that binds to and neutralizes IL-13), Anrukinzumab (IMA-638 and IMA-026) (Fully humanized $\operatorname{IgG}(1)$ antibodies that bind to different epitopes and neutralize IL-13 bioactivity), Lebrikizumab (IgG4 humanized monoclonal antibody that binds IL-13 with high affinity), Mepolizumab (Anti IL-5 humanized IgG1 monoclonal antibody: Awaiting FDA and EBM approval), Reslizumab (Humanized monoclonal anti-IL-5 antibody (IgG4/k)), Benralizumab (Humanized, Afucosylated monoclonal antibody against IL-5Ra), Brodalumab (Human IL-17RA-specific monoclonal antibody), Secukinumab (Anti-
IL-17 monoclonal antibody that selectively neutralizes IL-17A: Phase II ), Eculizumab (mAb (a hybrid of IgG2 and IgG4 Fc portion) cleaves and deactivates C5), Omalizumab (Blocks IgE to Fc-RI-Ig fusion protein and membrane Fc-RI: Approved FDA and EMA ). A trial of Anti-IgE monoclonal antibody in both adults and children with SPA is recommended [5].

Originally grown in Biotechnology, HMA has a number of qualities that made it attractive to early biotechnologist. HMA, which make up the vast majority of available biologic agents, are among the fastest-growing therapeutic biologics being developed to treat SPA. It yielded more than other drugs like receptor blockers. However, certain advantage such as have high target specificity; in fact, there are probably more advantages than disadvantages. This specific targeting minimizes the risks of side or adverse effects.

\section{References}

1. Yalcin AD, Celik B, Gumuslu S. D-dimer levels decreased in severe allergic asthma and chronic urticaria patients with the omalizumab treatment. Expert Opin Biol Ther 2014; 14: 283-286.

2. Yalcin AD. Advances in anti-IgE therapy. Biomed Res Int 2015; 317465.

3. Yalcin AD, Cilli A, Bisgin A, Strauss LG, Herth F. Omalizumab is effective in treating severe asthma in patients with severe cardiovascular complications and its effects on sCD200, d-dimer, CXCL8, 25-hydroxyvitamin D and IL-1 $\beta$ levels. Expert Opin Biol Ther 2013; 13: 1335-1341.

4. Mitchell PD, El-Gammal AI, O'Byrne PM. Emerging monoclonal antibodies as targeted innovative therapeutic approaches to asthma. Clin Pharmacol Ther 2016; 99: $38-48$.

5. Chung KF. Targeting the interleukin pathway in the treatment of asthma. Lancet 2015; 386: 1086-1096.

\section{*Correspondence to}

Arzu Didem Yalcin,

Allergy and Clinical Immunology,

Antalya Education and Research Hospital,

Antalya, Turkey 